

IJCRR

Section: Healthcare Sci. Journal Impact Factor: 5.385 (2017) ICV: 71.54 (2015)

\title{
Study of Prevelance and Pattern of Senserineural Hearing Impairment in Stage 5 Chronic Kidney Disease Patients on Haemodialysis- at a Tertiary Health Care Setup in India
}

\author{
Poulomi Saha ${ }^{1}$, Kapil Mondal' \\ 'RMO cum Clinical Tutor, Department of E.N.T. DR. B.C. ROY, PGIP Kolkata; ${ }^{2}$ Assistant Professor, Department of Medicine, Murshidabad \\ Medical College \& Hospital, Murshidabad, West Bengal.
}

\section{ABSTRACT}

Background: In patients with established CKD, multiple risk factors have been hypothesized to cause senserineural hearing loss. There is high rise in the prevalence of CKD in India due to rise in conditions such as diabetes and hypertension.

Aim of the Study: To identify the pattern of sensorineural hearing loss (SNHL) in stage 5 CKD patients on haemodialysis and to study the association between haemodialysis and degree of hearing changes.

Study Design: Case control, observational, analytical study.

Method: In this case control study 500 stage- 5 Chronic kidney disease adult patients on haemodialysis and 500 age and gender-matched healthy individuals were included over the period of 3 years. Upper age limit was restricted till 55 years to avoid presbyacusis. After base line investigations, they were subjected for all cases and controls were subjected otoscopic examination and hearing assessment using standard pure tone audiometry. Descriptive statistical analysis has been carried out in this study.

Result: In CKD group, $96.5 \%$ patients (5 patients had natural death) suffered from hearing loss and 3.5\%(17) patients had normal hearing. In control group, $31.2 \%$ suffered from Sensorineural hearing loss and $68.2 \%(343)$ have no hearing loss . Among the Stage 5 CKD group, $11.9 \%$ had mild SN hearing loss, $14.2 \%$ had moderate sensorineural hearing loss, $14.8 \%$ had moderately severe sensorineural hearing loss, $25.7 \%$ had severe SNHL, and $33.2 \%$ had profound SNHL. In the control group, $9 \%$ had mild SNHL, $7.6 \%$ had moderate SNHL, $7.1 \%$ had moderately severe SNHL,5.4\% had severe SNHL and $2.2 \%$ had profound SNHL. Deterioration of hearing acuity occurred in $62 \%$ of the patients at the end of follow-up and $12.4 \%$ of the participants showed improvement in hearing acuity. $25.6 \%$ patients showed no improvement.

Conclusion: Our finding in present study is that CKD patients with hearing loss received significantly lesser haemodialysis sessions is interesting as it suggests a possible beneficial association between increasing number of dialysis sessions and hearing loss.

Key Words: Haemodialysis, Hearing loss, Sensori-neural hearing loss

\section{INTRODUCTION}

Chronic kidney disease (CKD) refers to a gradual and usually irreversible reduction of glomerular filtration rate (GFR) which can be caused by trauma, genetic disorders, infections and effect of drugs that are toxic to the kidneys.
Other disease conditions such as diabetes and hypertension increase the risk of an individual developing kidney failure. About $10 \%$ of the world's population is affected by various forms of kidney disease making it a global public health challenge. The association between chronic kidney disease (CKD) and hearing impairment was first reported more than

\section{Corresponding Author:}

Kapil Mondal, Department of Medicine, Murshidabad Medical College \& Hospital, Murshidabad, West Bengal; Mob: 09635259541; E-mail: kapilmonda1980@gmai.com.

ISSN: 2231-2196 (Print)

Received: 10.12 .2019
ISSN: $0975-5241$ (Online)

Revised: 08.01 .2020
Accepted: 10.02 .2020 
80 years ago by Alport, who described a case of familial kidney disease related to hearing impairment. The etiopathogenetic mechanisms of Sensorineural hearing loss in CKD is due to osmotic alteration resulting in loss of hair cells, collapse of the endolymphatic space, edema and atrophy of specialized auditory cells and in some, complications of hemodialysis itself . Because of certain anatomic, physiological and ultrastructural similarities between the stria vascularis of the inner ear and basement membranes of glomeruli and evidence for similar antigenicity of the cochlea and kidney, may explain this association of CKD and SN loss can be explained to some extent. In patients with established $\mathrm{CKD}$, multiple risk factors have been hypothesized to cause hearing loss such as use of hypertension ototoxic drugs, hypertension, nephrotoxic drugs, electrolyte disturbances, diabetes, particularly in association with hypertension and hemodialysis itself and age of patient have been implicated in various studies. Despite the multitude of studies regarding hearing loss in CKD, some dark domains still persists regarding the role of haemodialysis and duration of disease. There is high rise in the prevalence of CKD in India due to rise in conditions such as diabetes and hypertension. Comparatively, hearing loss has been found to be more prevalent among CKD patients than the general population in different parts of the world ranging from $28 \%$ to $67 \%$ have been recorded in similar studies.

\section{OBJECTIVE}

1. To determine the pattern of sensorineural hearing loss (SNHL) in CKD patients on haemodialysis attending Medicine Department .

2. To study the association between haemodialysis and degree of hearing changes.

\section{STUDY DESIGN}

Case control, observational, analytical study

\section{MATERIALS AND METHODS}

Study area: Department of MEDICINE, Heamodialysis unit, Mursidabad Medical College, Berhampore. West Bengal.

Study period: November, 2016 - November, 2019.

Ethical clearance: Institutional ethics committee clearance was taken prior to the commencement of the study.

\section{Study sample}

Cases-500 CKD stage 5 (End Stage Renal Disease, ESRD) patients aged 18 to 50 years who have undergone at least one session of haemodialysis. Particularly, age was limited to 50 years for purposes of excluding presence of presbyacusis. CKD patients with conductive hearing loss and syndromic etiologies of CKD in which hearing loss is a known component, were excluded from this study.

Controls: As control group of 500, age and gender-matched healthy individuals were included in this study. These are the individuals without any history of ototoxic medications within the last three months, as well as those with no chronic diseases such as diabetes, hypertension, renal impairment and rheumatoid diseases, without family history of hearing loss, no prolonged noise exposure, no history of otosclerosis, other ear diseases and ear surgery.

\section{METHODOLOGY}

From all cases and controls documented informed consent was obtained

- All patients were investigated using standard performa containing details of age, gender, chronic illness, hypertension, diabetic status and past or recent administration of ototoxic medications.

- Duration of stage 5 chronic kidney disease and total number of haemoldialysis sessions obtained were documented.

- Both case and control groups were subjected to have complete haemogram, coagulogram, fasting and post prandial blood sugar, serum LFT and RFT, electrolytes, viral serology of HIV 1, HIV 2 and HBs Ag.

- All CKD patients and controls were subjected otoscopic examination and hearing assessment using standard pure tone audiometry at $250,500,1000$, 2000, 3000, 6000, 7000, and $8000 \mathrm{~Hz}$. As bone conduction threshold is limited upto $4000 \mathrm{~Hz}$,so the audiometric recordings $\geq 4000 \mathrm{~Hz}$ were done by air conduction testing only because High frequency sensorineural hearing recording by air conduction mode is unaffected by middle ear effusion. An average of the threshold levels of $>26 \mathrm{db}$ was considered as abnormal. A hearing loss of 26-40 db was classified as mild, $41-55 \mathrm{db}$ as moderate, $56-70$ as moderately severe, $71-90$ as severe, and $>90 \mathrm{db}$ as profound hearing loss. Air and bone conduction thresholds were compared to identify the type and degree of hearing loss.

Descriptive statistical analysis has been carried out in this study. Results on continuous measurements are presented on mean standard deviation (minimum and maximum) and results on categorical measurements are presented in number (\%). Chi square test is used to compare the difference in proportions. The significance is assessed at 5\% level of significance. Differences were considered significant if the $p$ value was less than 0.05 . 


\section{RESULT AND ANALYSIS}

\section{STAGES OF CKD}

\begin{tabular}{cc} 
Stage & GFR $\left(\mathrm{mL} / \mathrm{min} / \mathbf{1 . 7 3} \mathrm{m}^{2}\right)$ \\
0 & $>90$ \\
1 & 90 \\
2 & $60-89$ \\
3 & $30-59$ \\
4 & $15-29$ \\
5 & $<15$ \\
\hline
\end{tabular}

$\begin{aligned} & \text { World Health Organization classification of } \\
& \text { hearing loss }\end{aligned}$
\begin{tabular}{lc} 
Degree of hearing loss & dB hearing loss \\
Mild & $26-40$ \\
Moderate & $41-55$ \\
Moderately severe & $56-70$ \\
Severe & $71-91$ \\
Profound & $>91$ \\
\hline
\end{tabular}

Total 500 stage 5 chronic kidney disease patients were studied to measure the burden of sensorineural hearing loss in them. This was a case-control study. Five CKD patients succumbed to death in the course of the disease. They were excluded from the study. In the control group too one patient expired due to cerebrovascular accident (CVA).

After exclusion and inclusion criteria, among five hundred CRF control patients treated with hemodialysis, 357 were males and 143 females. Mean age in the case group was 51.9 for males and 42.5 for females. Duration of illness was $1-5$ years (mean 3.2). In control group 315 patients were male and 184 patients were female. Mean age in the control group was 50.3 for males and 41.2 for females.

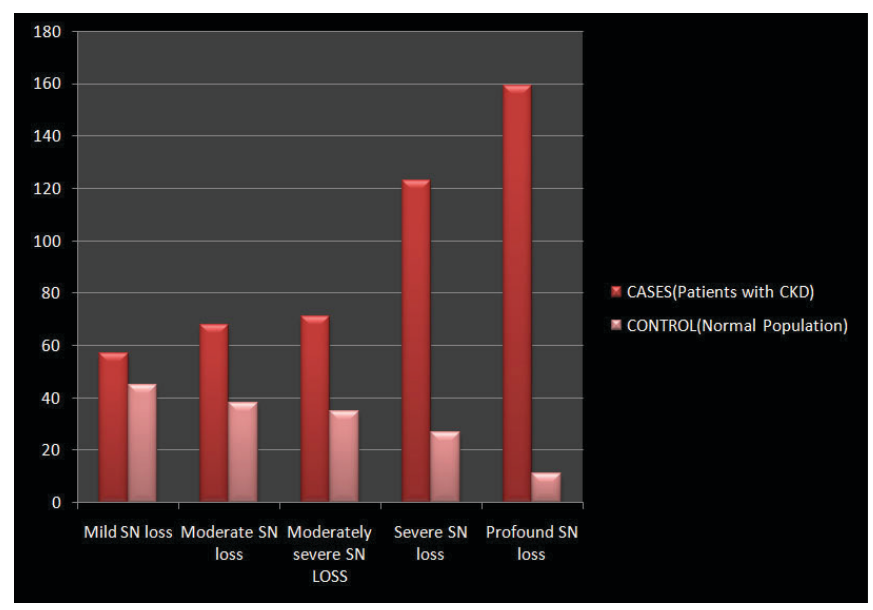

Diagram 1: Degree of SN hearing loss based on pure tone audiometry(PTA)
In CKD group, $96.5 \%$ patients (478 out of 495 ) suffered from hearing loss and $3.5 \%(17)$ patients had normal hearing. In control group, $31.2 \%$ (156 out of 499 patients) suffered from SN hearing loss and $68.2 \%(343)$ have no hearing loss .

Among the Stage 5 CKD group, 11.9\%(57 patients) had mild SN hearing loss, $14.2 \%$ had (68 patients)had moderate SNHL(sensori-neural hearing loss), $14.8 \%$ (71 patients) had moderately severe SNHL , 25.7\%(123 patients) had severe SNHL, and 33.2\%(159 patients) had profound SNHL.

In the control group, 9\% (45 people) had mild SNHL, 7.6\%(38 people) had moderate SNHL,7.1\% (35 people) had moderately severe SNHL, 5.4\% (27 people) had severe SNHL and 2.2\%(11 people) had profound SNHL.

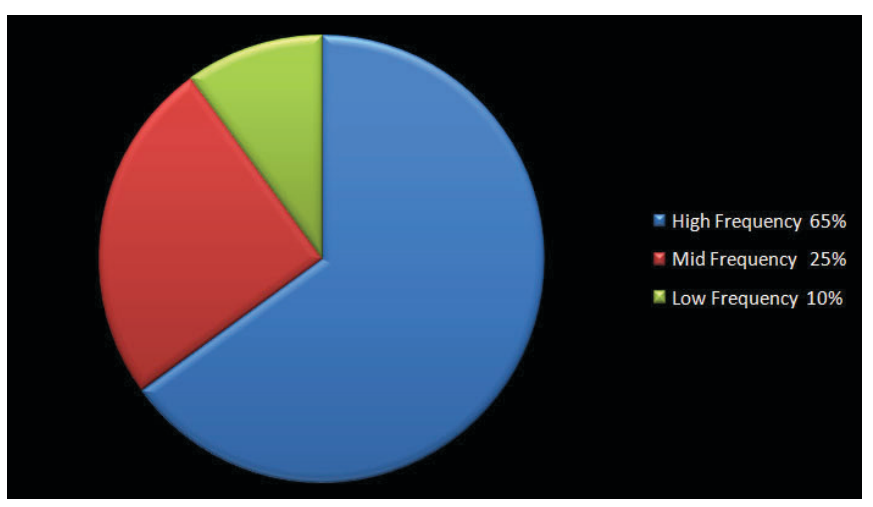

Diagram 2: Pattern of $S N$ hearing loss in Stage- 5 chronic kidney disease patients.

$65 \%$ Stage 5 CKD patients had high frequency $(8000 \mathrm{~Hz})$ SNHL(62 \% bilateral \& 3\% unilateral), $25 \%$ had mid frequency $(4000 \mathrm{~Hz})$ SNHL (19\% bilateral \& $6 \%$ unilateral) and $10 \%$ had low or at speech frequency $(500-2000 \mathrm{~Hz})$ SNHL ( $8 \%$ bilateral \& $2 \%$ unilateral).

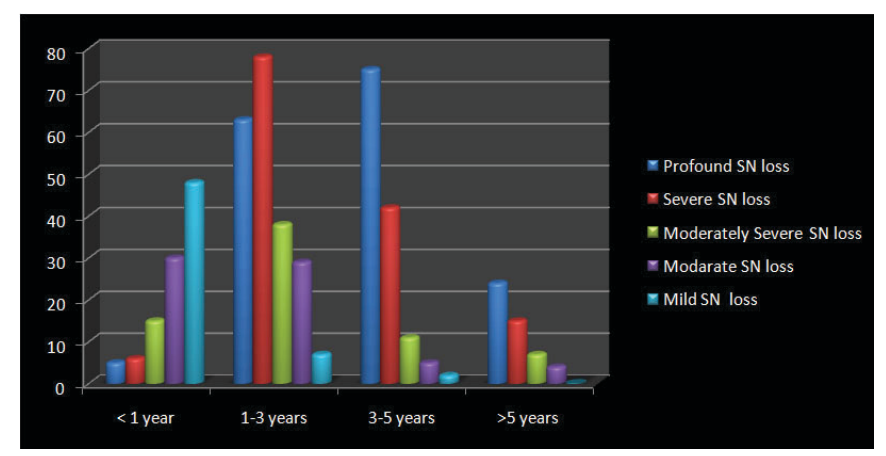

Diagram 3: Duration of disease among CKD patients with degree of hearing loss.

104 patients( $20.84 \%$ ) who were on haemodialysis for less than 1 year presented with hearing loss (hearing threshold above $20 \mathrm{~dB}$ ). 215 patients( $43.08 \%$ ) presented hearing loss 
who were on haemodialysis for more than 1 year but less than 3 years. The patients who were on haemodialysis for more than 3 years but less than 5 years were $135(27.2 \%$ ) in number . 50 patients $(10.1 \%)$ who were on haemodialysis for more than 5 years presented with hearing loss. The dominant hearing loss was obvious at high frequencies.

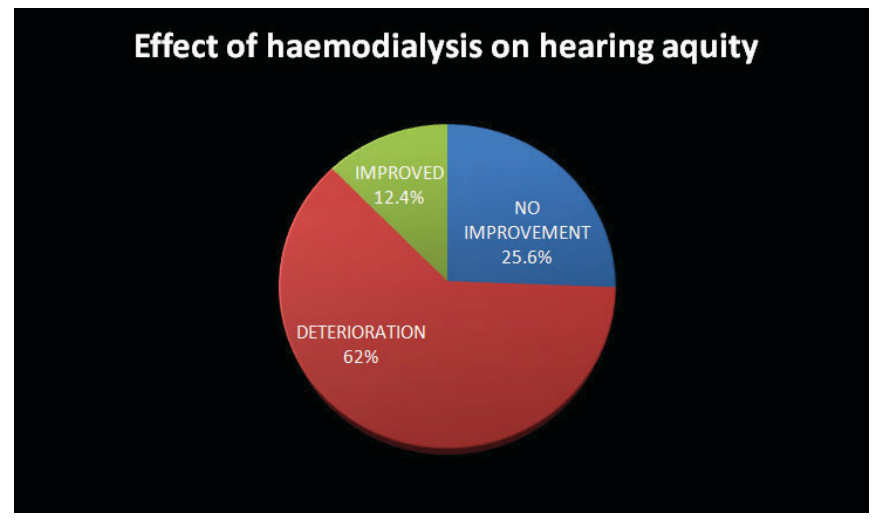

Diagram 4: Effects of hemodialysis on hearing acuity.

Deterioration of hearing acuity occurred in $62 \%$ of the patients at the end of follow-up and $12.4 \%$ of the participants showed improvement in hearing acuity. $25.6 \%$ patients showed no improvement.

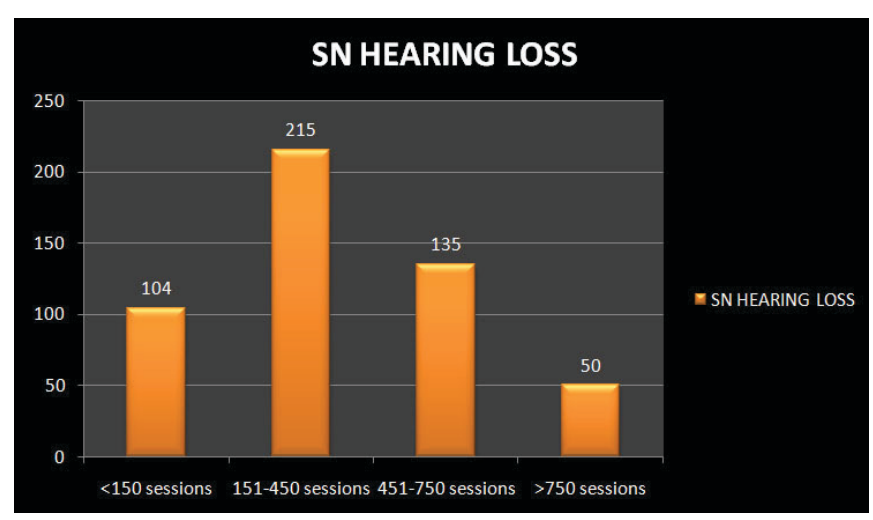

Diagram 5: Number of haemodialysis sessions in CKD patients with Senseri Neural Hearing loss.

Among CKD patients with hearing loss, the majority of the patients, 215 patients received 151 to 450 sessions. 104 patients received less than 150 sessions.135 patients received 451-750 sessions. 50 patients received more than 750 sessions.

\section{DISCUSSION}

A number of studies conducted in India and abroad have documented and published higher incidence of hearing loss among children and adults with chronic renal disease. The prevalence of hearing loss in CRF greatly varies in different countries. In India, it was reported to be $63.5 \%$, in Nigeria $67 \%$, in Iran $46 \%$, and in Croatia 63.6\% . Earlier studies showed higher prevalence of hearing loss even upto $70 \%-75 \%$. This variation could be due to differences in the age of patients, methods of assessment of hearing loss, or duration of CRF and hemodialysis.

The exact pathophysiology between hearing impairment and CKD is addressed in various ways. Over many years', research have linked $\mathrm{CRF}$ and hearing loss in patients with rare diseases like mitochondrial myopathy, lactic acidosis, stroke, Alport syndrome and Fabry's disease.

Electrolyte disturbances, particularly sodium, water imbalance, hypertension, Vitamin D deficiency, and elevated serum urea levels are some proposed mechanisms for hearing impairment in patients with CRF. Defects in the cationic gradient of endolymphatic fluid can adversely affect hearing. Along with this alterations in the peripheral and central nervous system, "uremic neuropathy," may lead to hearing impairment in CRF. Di Paolo et al reported a high incidence of nerve conduction dysfunction in patients with CRF. They found decreased conduction velocity in the sensory and motor units, with the sensory units being more compromised than the motor.

Rossini et al were unable, to arrive at a definite conclusion regarding the effect of haemodialysis on hearing in CKD. In contrast, Peyvandi et al proposed in a recent study that prevalence and severity of hearing loss increases with duration of CKD and haemodialysis.

Ozen et al reported audiometric improvement of 20 decibel of hearing perception of chronic kidney disease patients following haemodialysis. They hypothesized that haemodialysis causes change in serum osmolarity, BUN and fluid retention which may reverse the hearing impairment in post dialysis period. Though contradicting studies like are there questioning hearing improvement following haemodialysis.

Contributory evident data to indicate beneficial role of haemodialysis on hearing threshold in CKD patients is present in this study. Possible beneficial association between increasing number of dialysis sessions and hearing threshold improvement is documented in $12.4 \%$ patients of chronic kidney disease because we found that chronic kidney disease patients with hearing loss received significantly lesser haemodialysis sessions in comparison to those having higher number of haemodialysis sessions. We concluded the hearing threshold improvement occurred due to improvement of serum osmolality following haemodialysis. Improved serum osmolality may have reverted the collapse of endolymphatic space and may have reduced the edema of some functionally 
active auditory cells. Thus causing improvement of hearing threshold to some extent.

In our study deterioration of hearing occurred in $62 \%$ of the patients at the end of follow-up. $12.4 \%$ of the participants showed improvement in hearing acuity. 25.6\% patients showed no improvement.

We also observed that hearing loss at high frequencies might be the most common audiometric abnormality in CKD patients, but not the typical one. This audiometric difference, that is the presence of high frequency, mid frequency and even low frequency SN loss in CKD may be due to several reasons. But typical detection of high frequency in the above studies were mostly due to small sample sizes in both studies(49 and 63 CKD patients, respectively). Secondly, the questions were only used to access high-frequency SN loss in audiometry in patients, possibly not designing questionnaire for patients with mild and moderate frequency $\mathrm{SN}$ loss in conventional audiometry.

So these number of studies clearly established prevalence of hearing loss among CKD patients, but well-designed studies with larger sample sizes were needed to adress relationships between hearing loss in CKD and haemodialysis. Also another important question that arises in this context is whether a blanket audiometric screening of all CKD patients would help identify mild hearing loss and prevent progression to severe degrees, especially if a beneficial role for haemodialysis can be definitely established in long-term prospective studies. This present study answers both of the questions to some extent.

This study found $96.5 \%$ (478 patients) of CKD patients on haemodialysis had hearing loss, considerably higher than the normal population who had $31.2 \%$ hearing loss [FIGURE: 1]. In other words, mostly all stage 5 CKD patient on haemodialysis experiences some degree of hearing impairment. 11.9\%(57 patients) had mild SN hearing loss, $14.2 \%$ had (68 patients)had moderate SNHL(sensorineural hearing loss), $14.8 \%$ (71 patients) had moderately severe SNHL , 25.7\% (123 patients) had severe SNHL, and $33.2 \%$ (159 patients) had profound SNHL. That is $40.5 \%$, majority of the patients had moderately severe to severe SN loss. Hearing thresholds were abnormal in CKD patients on haemodialysis across all frequencies ranging from $250 \mathrm{~Hz}$ to $8 \mathrm{kHz}$. SN loss was high frequency in $65 \%$ patients, mid frequency in $25 \%$ patients and low frequency in $10 \%$ patients [FIGURE: 2]

The prevalence of hearing loss in CKD patients varies from 28 per cent to 77 per cent according to different studies. Notably, many older studies have shown higher prevalence of hearing loss, possibly due to small sample sizes. The prevalence of this study is $\mathbf{4 8 . 9 \%}$.
The high prevalence of hearing loss at enrollment in the study reflects the significant effect of CRF on hearing function. Yet, the impact of hemodialysis on the hearing threshold after 18 months was still substantial. The hearing threshold deteriorated from $30.5 \pm 15.1 \mathrm{~dB}$ at the start of the study to $45.2 \pm 11.3 \mathrm{~dB}$ after 18 months, which was a highly significant difference $(P<0.001)$. This study also showed that CKD patients with hearing loss received [ FIGURE: 5 ]

significantly fewer haemodialysis sessions compared to those without hearing loss. Our finding that CKD patients with hearing loss received significantly fewer haemodialysis sessions is interesting as it suggests a possible beneficial association between increasing number of dialysis sessions and hearing loss.

Thus, it is evident that there are data to indicate contributory as well as beneficial roles for haemodialysis on hearing in CKD patients. The finding of Lasisi et al. supports this result. The role of hemodialysis in the occurrence of hearing loss among patients with CRF could be due either to changes in the fluid and electrolyte composition of endolymph or accumulation of amyloid materials in inner ear tissues. Aluminum toxicity associated with chronic dialysis may play a role in hearing loss.

Nikolopoulos et al, Ozturan and Lam, Stavroulaki et al, and Pandey et al showed that auditory functions and thus hearing acuity were not affected by hemodialysis, particularly short term dialysis, or at least in the first 5 years of treatment. In contrast, other researchers reported that hearing is improved by hemodialysis. Aspris et al indicated that although there is improvement in neural auditory function following hemodialysis, it is not restored to a normal level. Gafter et al. concluded that although dialysis may have some temporary beneficial effect, the longterm effect is not assured. The acceptable explanation of hearing improvement particularly in low frequency hearing loss is that hemodialysis promotes normalization and stabilization of hydroelectric and metabolic changes in the endolymph that were induced by CRF, leading to enhancement of neural conduction and restore hair cell function.

\section{CONCLUSION}

Mild sensorineural hearing impairment in CKD patients on haemodialysis is well documented in number of studies. Though this hearing loss is mostly high frequency but other emerging studies marks significant presence of low frequency and mid frequency loss .These patients were likely to be older and had a significantly longer exposure to ototoxic drugs. Haemodialysis might have a promising effect on hearing loss in CKD patients, but further research by long-term prospective studies are required to link common pathophysiology between kidney dysfunction and cochlear 
impairment to potentially modify the normal care of CKD patients of higher stages. It should encourage clinicians to regularly question about hearing function in their preventive care protocols and to refer all CKD patients with hearing loss to otorhinolaryngologists for evaluation and rehabilitation .

\section{ABBREVIATION:}

1. $\mathrm{CKD}=$ Chronic kidney disease

2. $\mathrm{CRF}=$ Chronic renal failure

3. $\mathrm{SNHL}=$ Senserineural hearing loss

4. $\mathrm{ESRD}=$ End stage renal disease

5. $\mathrm{LFT}=$ Liver function test

6. $\mathrm{RFT}=$ Renal function test

7. $\mathrm{Hz}=$ Hertz

8. $\quad \mathrm{CVA}=$ Cerebro vascular accident

9. $\mathrm{Db}=$ Decibel

$\mathrm{BUN}=$ Blood urea nitrogen.

\section{REFERENCES}

1. Joseph O. Boateng et al Hearing impairment among chronic kidney disease patients on haemodialysis at a tertiary hospital in Ghana. Ghana Medical Journal. Volume 53 Number 3 September 2019; 53(3): 197-203.

2. C Balasubramanian et al A Study of Hearing Loss in Chronic Renal Failure. International Journal of Scientific Study | January 2018 | Vol 5 | Issue 10. Print ISSN: 2321-6379.Online ISSN: 2321-595X.

3. Haider K. Saeed et al Sensorineural hearing loss in patients with chronic renal failure on hemodialysis in Basrah, Iraq. Tzu Chi Medical Journal 2018; 30(4): 216-220.

4. E. K. Reddy et al Proportion of hearing loss in chronic renal failure: Our experience. 2016, Indian Journal of Otology. 22:4-9. IP: [110.227.81.227]

5. Jishana Jamaldeen et al Prevelence and pattern of hearing loss chronic kidney disease patients undergoing haemodialysis. Australian Medical Journal. [AMJ] 2015;8(2):41-46.

6. Young Joon Seo et al Association of hearing impairment with chronic kidney disease: a cross-sectional study of the Korean general population. BMC Nephrology. 2015. 16:154 .DOI 10.1186/s12882-015-0151-0.

7. Somashekara KG et al Etiological evaluation of hearing loss in chronic renal failure. Indian Journal of Basic and Applied Medical Research, 2015; 4(2):194-199.
8. Sahin C \& Varim C. Hearing loss in geriatric hemodialysis patients. Med J DY Patil Univ, 2015; 8:734-8.

9. Peyvandi A et al Hearing loss in chronic renal failure patient undergoing hemodialysis. Indian J Otolaryngol Head Neck Surgery 2013 Dec;65(Suppl 3):537-40.

10. Klagenberg KF et al Vestibular dysfunction in adolescents and young adults after kidney transplant. Int Tinnitus J. 2013; 18(2):149-155.

11. Meena RS et al Hearing loss in patients of chronic renal failure: a study of 100 cases. Indian J Otolaryngol Head Neck Surg. 2012 .Dec;64(4):356-9.

12. Adekwu A et al Prevalence of hearing loss in chronic kidney disease, stages 3-5 patients in a teaching hospital in Nigeria. J Med Res Pract, 2012; 1(2): 74-76.

13. Sharma $R$ et al A study on hearing evaluation in patients of chronic renal failure. Indian J Otol. 2011; 17: 109-12.

14. Pandey $\mathrm{S}$ et al Audiometric profile in patients with chronic renal failure. J Otolaryngol Head Neck Surg 2011;40:131-6.

15. Vilayur $\mathrm{E}$ et al The association between reduced GFR and hearing loss: a cross-sectional population-based study. Am J Kidney Dis. 2010 Oct;56(4):661-9.

16. Jakic $M$ et al Sensorineural hearing loss in hemodialysis. patients. Coll Antropol. 2010 Mar;34 Suppl 1:165-71.

17. Lasisi AO et al Hearing threshold in patients with chronic renal failure. Saudi Med J. 2007 May;28(5):744-6.

18. Bains $\mathrm{KS}$ et al Cochlear function in chronic kidney disease and renal transplantation: A longitudinal study. Transplant Proc 2007; 39:1465-8.

19. Lasisi AO, Salako BL, Kadiri S, Arije A, Oko-Jaja R, Ipadeola A, Olatoke F. Sudden sensorineural hearing loss and heamodialysis. Ear, Nose \& Throat Journal,2006; 85: 819-821.

20. Thodi $\mathrm{C}$ et al Hearing in renal failure. Nephrol Dial Transplant 2006;21:3023-30.

21. Qin Y et al Application of extended high frequency audiometry in the patients with chronic renal failure treated by hemodialysis. Lin Chuang Er Bi Yan Hou Ke Za Zhi 2005;19:17-8.

22. Zeigelboim BS et al High frequency audiometry and chronic renal failure. Acta Otolaryngol 2001;121:245-8.

23. Serbetçioglu MB et al Effects of a single session of hemodialysis on hearing abilities. Acta Otolaryngol 2001;121:836-8.

24. Stavroulaki $P$ et al Hearing evaluation with distortion-product otoacoustic emissions in young patients undergoing haemodialysis. Clin Otolaryngol Allied Sci 2001;26:235-42.

25. Hutter JC et al Acute onset of decreased vision and hearing traced to hemodialysis treatment with aged dialyzers. JAMA 2000;283:2128-34. 